\title{
Rotation axis estimation for the derotator calibration with machine vision measurement of the auxiliary laser
}

Yin, Yongkai, Altmann, Bettina, Pape, Christian, Reithmeier, Eduard

Yongkai Yin, Bettina Altmann, Christian Pape, Eduard Reithmeier, "Rotation axis estimation for the derotator calibration with machine vision measurement of the auxiliary laser," Proc. SPIE 10819, Optical Metrology and Inspection for Industrial Applications V, 108190S (2 November 2018); doi: 10.1117/12.2500549

SPIE. Event: SPIE/COS Photonics Asia, 2018, Beijing, China 


\title{
Rotation axis estimation for the derotator calibration with machine vision measurement of the auxiliary laser
}

\author{
Yongkai Yin*a, b, Bettina Altmann ${ }^{\mathrm{a}}$, Christian Pape ${ }^{\mathrm{a}}$, Eduard Reithmeier ${ }^{\mathrm{a}}$ \\ anstitute of Measurement and Automatic Control, Leibniz University Hannover, Nienburger Str.17, \\ D-30167 Hannover; ${ }^{b}$ Shandong Provincial Key Laboratory of Laser Technology and Application, \\ School of Information Science and Engineering, Shandong University, Jinan 250100, China
}

\begin{abstract}
A key problem when using the derotator is to ensure the coaxial alignment between the optical axis of the derotator and the rotation axis of the measured object. Moreover, accurate measurement of the rotation axis is the prerequisite of achieving the coaxial alignment. In this paper, we propose a strategy for estimation of the rotation axis with the assistant of an auxiliary laser and a binocular stereo vision sensor. When a laser source is temporarily attached to the measured object or the rotor shaft, the rotation of the laser will generate a ruled surface. The binocular sensor can accurately measure the coordinates of laser dots in three-dimensional (3D) space. The pose vector of the laser in each certain rotation angle can be estimated with measured 3D coordinates of the laser dots. Finally, the rotation axis of the object can be estimated with multiple pose vectors considering the constraint of the fixed axis rotation. The validity and precision of this proposed strategy are demonstrated by means of experiments.
\end{abstract}

Keywords: rotation axis estimation, binocular stereo vision, derotator calibration, least squares fitting, auxiliary laser, 3D measurement

\section{INTRODUCTION}

An optomechanical image derotator is a device, which can compensate for the image blur caused by the rotation of the object, as shown in Fig. 1. The derotator has a wide range of potential applications in dynamic inspection ${ }^{1,2}$ and vibration monitoring ${ }^{3,4}$ of objects with high rotational speeds. The core component of the derotator is a Dove $\mathrm{e}^{5}$ or reflector prism ${ }^{6}$. When the prism rotates at half the rotational speed of the object, the image transmitted through the prism will appear stationary. To guarantee the stationary image, a key problem when using a derotator is to ensure the coaxial alignment between the optical axis of the derotator and the rotational axis of the measured object.

To achieve coaxial alignment, the mathematical model between the movement of the image and the misalignment of the two axes is established, in which the trajectory of a marker can be described with the limaçon of Pascal ${ }^{7,8}$. The parameters of the limaçon correlate with the magnitude of the misalignment. The parameters can be minimized by adjusting the pose of the derotator to achieve the coaxial alignment. However, this model cannot distinguish the shift and the tilt between the two axes ${ }^{7}$, thus a procedure to make the two axes parallel is need.

\footnotetext{
*yinyongkai@gmail.com
}

Optical Metrology and Inspection for Industrial Applications V, edited by Sen Han, Toru Yoshizawa, Song Zhang, Proc. of SPIE Vol. 10819, 108190S · @ 2018 SPIE CCC code: $0277-786 \mathrm{X} / 18 / \$ 18 \cdot$ doi: $10.1117 / 12.2500549$ 


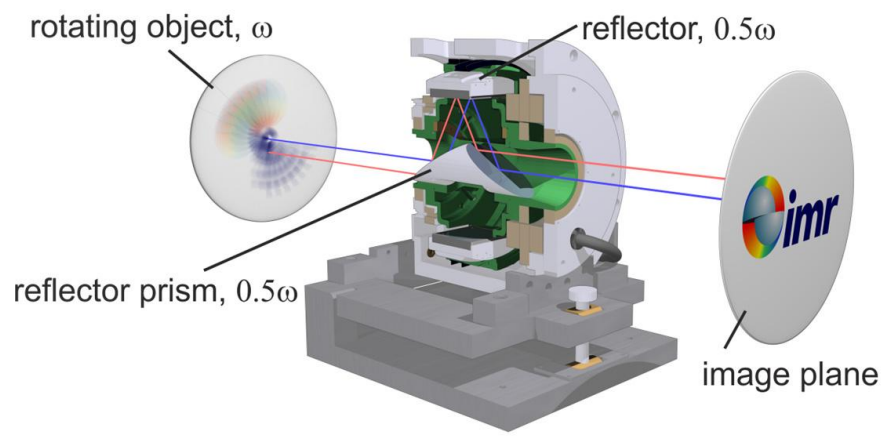

Figure 1. The image derotator

An alternative approach to achieve the coaxial alignment is adjusting the pose of the derotator directly based on the transformation matrix between the two axes. To calculate the transformation matrix accurately, the rotation axis of the object should be measured in three-dimensional (3D) Euclidean space. In this paper, a strategy providing rotation axis estimation is proposed with the assistant of an auxiliary laser and a binocular stereo vision system. When a laser source is temporarily attached to the measured object or the rotor shaft, the rotation of the laser will generate a ruled surface. The binocular sensor can accurately measure the coordinates of laser dots in three-dimensional (3D) space. The pose vector of the laser in each certain rotation angle can be estimated with measured 3D coordinates of the laser dots. Finally, the rotation axis of the object can be estimated with multiple pose vectors considering the constraint of the fixed axis rotation. Details of the proposed approach are described in section 2, the verification experiments and result analysis are presented in section 3 , and section 4 is the conclusion.

\section{PRINCIPLE}

A binocular stereo vision sensor is employed to estimate the parameters of axis quantitatively and to evaluate them easily. The object and the derotator are usually separated by a relatively large distance. However, the rotation axes of both, the derotator and the object, are expected to be measured in the same coordinate system. Since the binocular sensor has limit measurable volume, an auxiliary laser is introduced to make sure the measurement for the rotation axis of the object can be achieved at any distance from the object. The proposed approach is schematically shown in Fig. 2. When a laser source is temporarily attached to the rotating object, the rotation of the laser line will generate a ruled surface. Generally, the ruled surface is a hyperboloid. Only when the laser exactly intersects with the rotation axis of the derotator, the surface will become a cone. The rotation axis of the object is just the symmetry axis of the ruled surface. Therefore, the rotation axis can be estimated with multiple laser lines at the different angle. It is obviously that the parameters of each line should be estimated in advance. Technically, a line in 3D space can be fitting with multiple points on it. In addition, when an opaque plate is placed in the path of laser, there will be a laser dot resulting from the intersection between the laser line and the plate. Changing the position of the plate along the direction of the laser can get different dots on the laser line. 


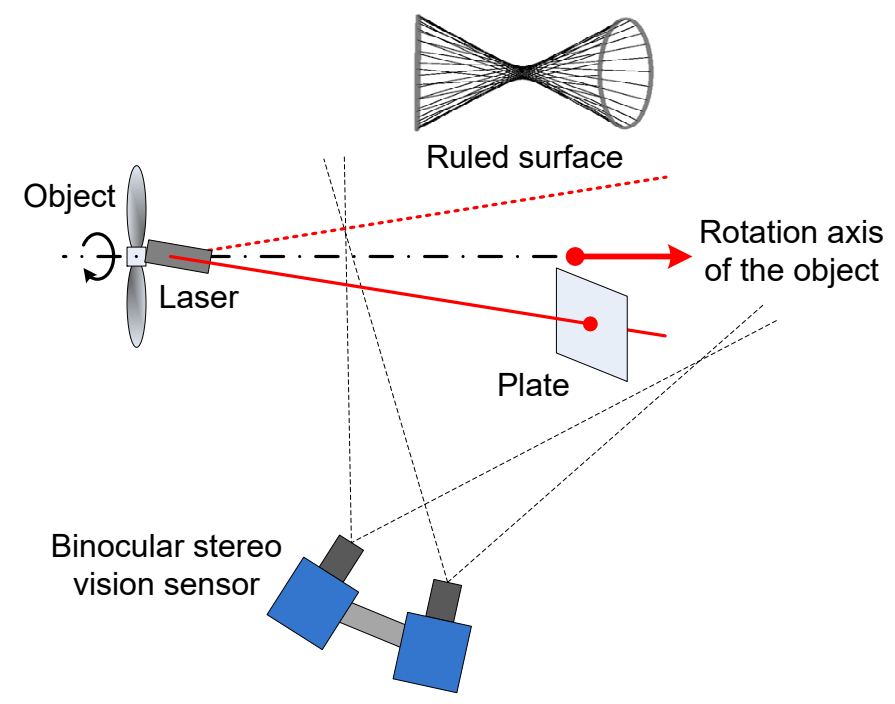

Figure 2. Schematic diagram of the proposed approach for rotation axis estimation with the binocular stereo vision sensor and the auxiliary laser

\subsection{Laser dot location}

First of all, a binocular stereo vision sensor is established to measure the coordinates of laser dots in 3D space. By using binocular sensor, with the image coordinates of the laser $\operatorname{dot} \hat{\mathbf{x}}$, the 3D coordinates of the laser dot $\mathbf{X}$ can be calculated with the principle of binocular stereo vision ${ }^{9}$

$$
\left\{\begin{array}{l}
s_{l} \tilde{\mathbf{x}}_{l}=\mathbf{K}_{l} \mathbf{X} \\
\hat{\mathbf{x}}_{l}=\mathbf{x}_{l}+\delta\left(\mathbf{k}_{l}\right) \\
s_{r} \tilde{\mathbf{x}}_{r}=\mathbf{K}_{r}[\mathbf{R X}+\mathbf{t}] \\
\hat{\mathbf{x}}_{r}=\mathbf{x}_{r}+\delta\left(\mathbf{k}_{r}\right)
\end{array}\right.
$$

where $\tilde{\mathbf{x}}$ denotes the homogeneous coordinate, $\mathbf{K}$ is the intrinsic parameter matrix, $\delta(\mathbf{k})$ is the lens distortion parameterized by the distortion coefficients $\mathbf{k}$, and $s$ is a scale factor. The subscript $l$ and $r$ denote the left and right camera in the binocular sensor respectively, while the rotation matrix $\mathbf{R}$ and the translation vector $\mathbf{t}$ are the structure parameters between the two cameras. Here the coordinate system of the left camera is chosen as the coordinate system of the sensor. All the parameters of the sensor $\left(\mathbf{K}_{l / r}, \mathbf{k}_{l / r}, \mathbf{R}, \mathbf{t}\right)$ should be estimated in advance with appropriate calibration algorithms ${ }^{10}$.

The center coordinates of the laser $\operatorname{dot} \hat{\mathbf{x}}=\left(c_{u}, c_{v}\right)^{\mathrm{T}}$ is extracted from the image containing the laser dot. Due to the high intensity and good focusing ability of the laser, the laser dot has a sharp peak in its intensity distribution. Therefor the subpixel center of the laser dot can be calculated with a classical centroid algorithm

$$
c_{u}=\frac{\sum u I(u, v)}{\sum I(u, v)}, c_{v}=\frac{\sum v I(u, v)}{\sum I(u, v)}
$$


where $(u, v)$ are the image coordinates of the pixel, and $I(u, v)$ is the corresponding intensity of the pixel.

\subsection{Laser line fitting}

Generally, a line $\mathbf{l}$ in 3D space can be expressed with

$$
\mathbf{I}=\mathbf{p}+\lambda \mathbf{n}
$$

where $\mathbf{p}$ is a certain point on the line and $\mathbf{n}$ is the direction vector of the line. The variation of the coefficient $\lambda$ will lead to point traversal along the line. To fit a line with 3D point set $\left\{\mathbf{X}_{i}, i=1, \cdots, N\right\}, \mathbf{p}$ and $\mathbf{n}$ should be estimated separately. First, the point $\mathbf{p}$ can be estimated by calculating the mean of the point set. Then the point set can be centralized and the direction $\mathbf{n}$ can be calculated with the singular value decomposition (SVD) algorithm.

\subsection{Rotation axis estimation}

Essentially, the rotation axis of the object $\mathbf{a}$ is also a line in 3D space, so it can be described with the similar expression in Eq. (3)

$$
\mathbf{a}=\mathbf{q}+\lambda \mathbf{v}
$$

where $\mathbf{q}$ is a certain point on the axis and $\mathbf{v}$ is the direction vector of the axis. As mentioned above, the rotation of the laser will generate a ruled surface. Fortunately, whether it is the hyperboloid or the cone, the surface ultimately comes from the rotation of a line around a fixed axis. Thus, there is a fixed angle $\theta$ between the laser line $\mathbf{I}$ and the rotation axis $\mathbf{a}$, which results in the following constraint

$$
\angle(\mathbf{v}, \mathbf{n})=\theta
$$

Considering this constraint, the direction vector of the axis $\mathbf{v}$ can be estimated with the following optimization

$$
\arg \min _{\mathbf{v}, \theta} \sum_{j=1}^{M}\left(\angle\left(\mathbf{v}, \mathbf{n}_{j}\right)-\theta\right)^{2}
$$

It should be notice that $\theta$ is also unknown, thus it should be estimated together with $\mathbf{v}$.

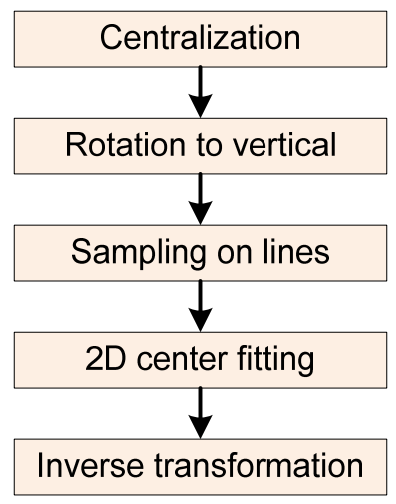

Figure 3. The flow chart of the position estimation for the rotation axis 
The flow chart of the estimation for the position point $\mathbf{q}$ is shown in Fig. 3. Multiple lines corresponding to different rotation angle of the laser are exploited to the following process. First, the positions of all the lines are centralization with their mean coordinates. Then the coordinate system is rotated to make the direction $\mathbf{v}$ parallel with the $z$-axis. This rotation makes sure all the lines have the same angle relative to the $x-o-y$ plane. The intersections between each line and the plane $z=z_{s}$ are calculated and will be used for the center fitting later. A benefit of the previous rotation is that the distribution of the intersection is now a circle on the horizontal plane. Thus, the center of the circle $\left(x_{c}, y_{c}\right)$ can be estimated with 2D circle fitting. Multiple sets of the intersection can be sampled by changing the value of $z_{s}$. The center fitting algorithm is applied to each intersection set and the mean center $\left(\bar{x}_{c}, \bar{y}_{c}\right)$ is calculated to improve the reliability of the estimation. In the current coordinate system, the position point is now $\mathbf{q}^{\prime}=\left(\bar{x}_{c}, \bar{y}_{c}, z_{c}\right)^{\mathrm{T}}$, where $z_{c}$ can take any value since the direction of the rotation axis is along the $z$-axis. Here $z_{c}$ is set to zero with the consideration of the previous centralization. Finally, the estimated center $\left(\bar{x}_{c}, \bar{y}_{c}, 0\right)$ is transformed back to the original coordinate system by inverse rotation and center translation.

\subsection{The complete process}

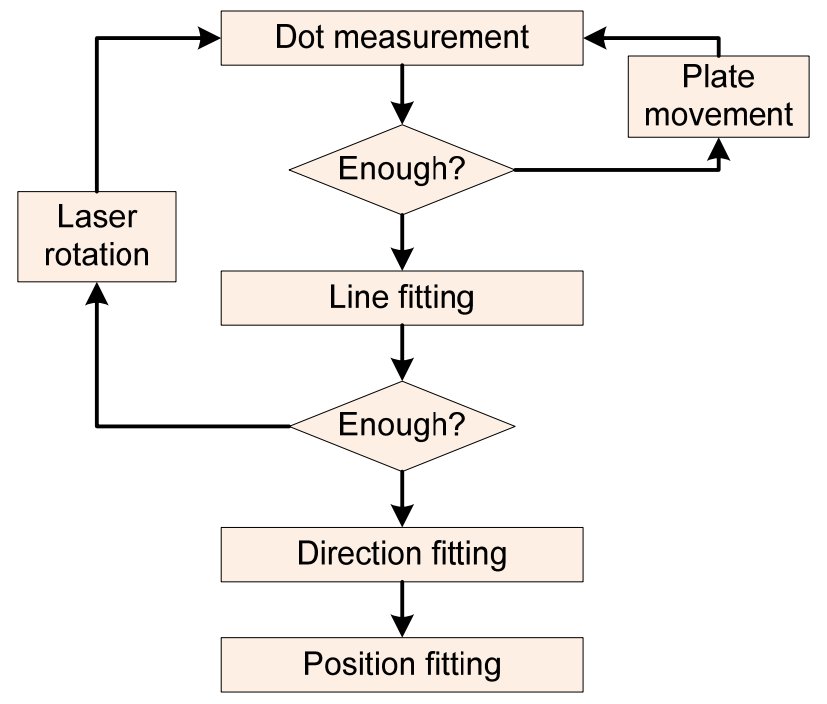

Figure 4. The flow chart of the rotation axis estimation

The complete flow chart of the rotation axis estimation is schematically shown in Fig. 4. First of all, the 3D coordinates of the laser dot are measured with the binocular stereo vision sensor. The plate to receive the laser dot should be moved along the direction of the laser to get different dots on the laser line. Then the parameters of current line can be estimated with the multiple dots. In addition, the laser should also be rotated to different angles so that multiple lines around the rotation axis can be measured. Finally, the rotation axis can be estimated with the parameters of those multiple lines. The direction vector is calculated with the fixed angle constraint, and then the position point can be estimated with points form the multiple lines. 


\section{EXPERIMENTS}

\subsection{Experimental setup}

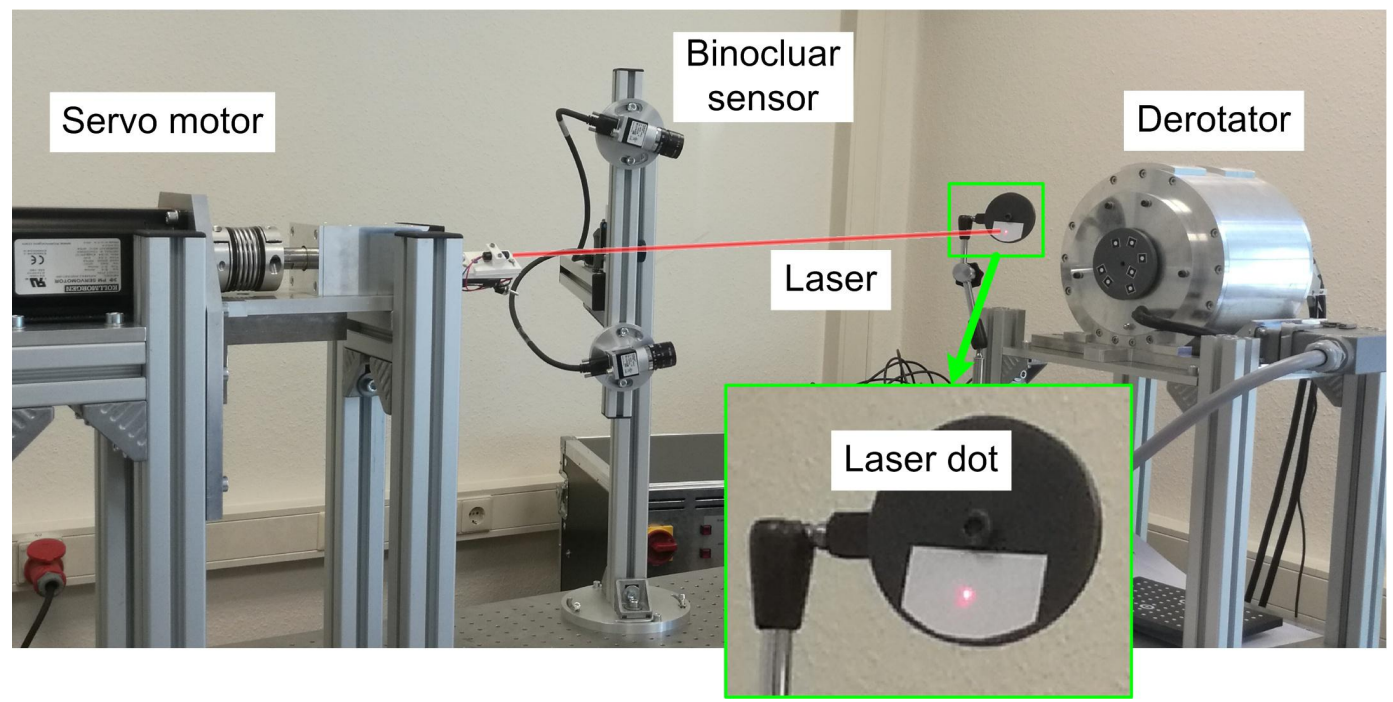

Figure 5. The experimental setup

The experimental setup shown in Fig. 5 is used for a series of experiments to verify the proposed approach. The binocular stereo vision sensor consists of two monochrome cameras (Basler acA1300-200um, $1280 \mathrm{px} * 1024 \mathrm{px}$ ) whose lenses have a focal length of $16 \mathrm{~mm}$ (RICOH FL-CC1614-2M). A planar target with the pattern of a dot matrix is employed to calibrate the sensor. The calibration use a conventional strategy, in which the left and right cameras are first calibrated separately and then all the parameters of the stereo vision sensor are optimized together. The pattern of the calibration target is printed with an ordinary laser printer. To eliminate the errors from the inaccurate target pattern, the coordinates of all the dots on the target are refined with the bundle adjustment technique before the sensor calibration.

\subsection{Experiments and analysis}

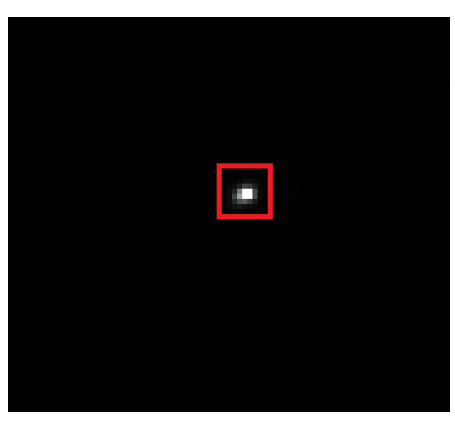

(a)

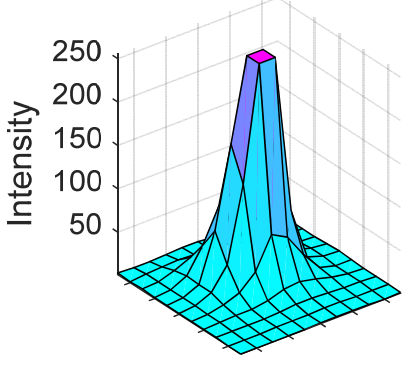

(b)

Figure 6. The data of a laser dot with an exposure time of $200 \mu$ s. (a) The captured image with dark background; (b) The wireframe mesh of the local intensity distribution around the laser dot. 
The well-focused laser has a very high intensity. To capture the image of the laser dot with good quality, a short exposure time is recommended to avoid the over-exposure and aperture diffraction. Another benefit from the short exposure time is that the background of the whole image has a quite low intensity, thus a simple threshold segmentation algorithm can detect the laser dot correctly. An image of the laser dot with an exposure time of $200 \mu$ s is shown in Fig. 6(a). The background is almost completely black. Moreover, the wireframe mesh of the local intensity distribution within the red square is shown in Fig. 6(b), where the sharp peak is employed to calculate the centroid of the laser dot with subpixel accuracy.

Some typical results of different steps in the rotation axis estimation are shown in Fig. 7. Figure 7(a) is the result of line fitting, where the magenta points are the measured laser dots, and the cyan arrow is the estimated direction $\mathbf{n}$. The start point of the cyan arrow is determined by the estimated position point $\mathbf{p}$. Figure 7(b) is the result of direction fitting, where the cyan arrows are the direction vector of different laser lines, and the red arrow is the estimated direction vector $\mathbf{v}$ of the rotation axis. Figure 7(c) is a collective display of the axis estimation with all the data, where the red arrow also denotes the direction of the axis $\mathbf{v}$ while the start point of the red arrow is determined by the estimated position point $\mathbf{q}$.

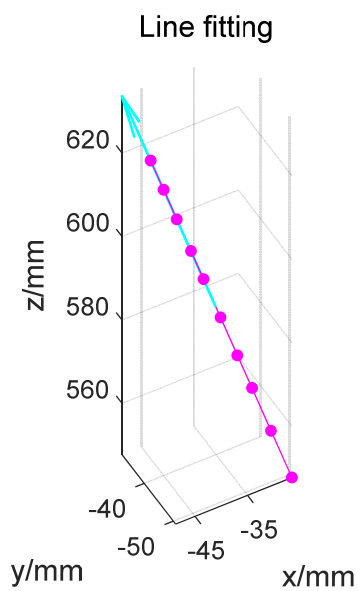

(a)

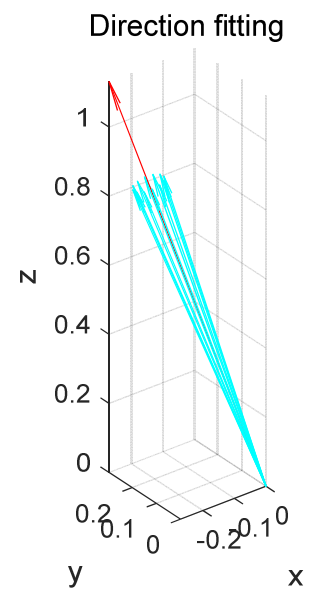

(b)

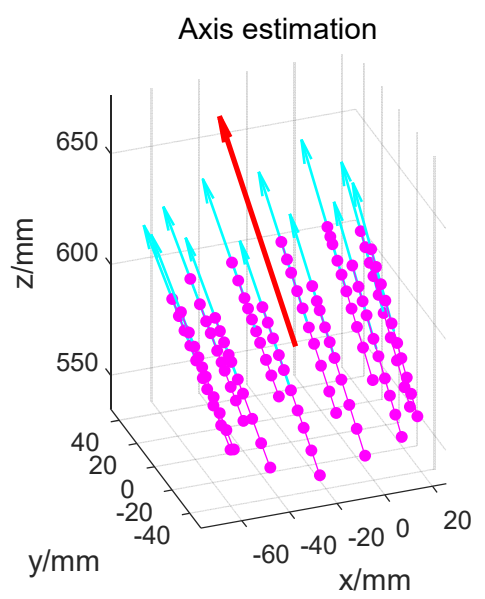

(c)

Figure 7. Plotting display of different steps in the axis estimation. The magenta points are the measured laser dots, while the cyan arrows are the direction vectors of different laser lines, and the red arrow is the direction vector of the rotation axis. (a) Line fitting with multiple measured laser dots; (b) Direction fitting of the rotation axis with multiple estimated line directions; (c) The result of the axis estimation with all the data.

When the performance of the proposed approach is evaluated, only measurement precision but not accuracy can be considered, because there is no reference axis with truth-values of the parameters. Here the precision of line fitting is demonstrated with 30 measurements of the stationary laser line. The position point $\mathbf{p}$ will change with the position of the measured laser dots, thus only the variation distributions of the direction vector $\mathbf{n}$ are shown in Fig. 8. The standard deviation (STD) for the polar angle of the estimated directions is $0.0131^{\circ}$, and for the azimuthal angle is $0.0394^{\circ}$. 

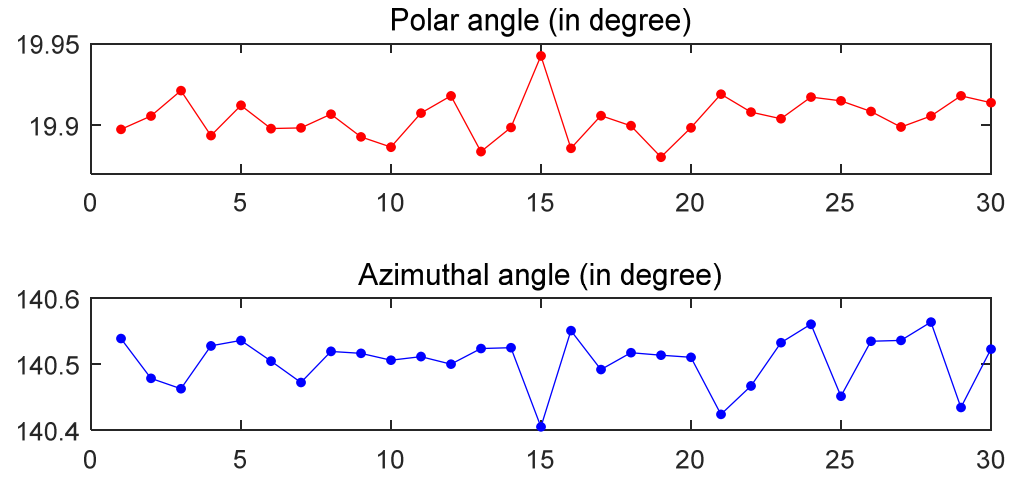

Figure 8. The variation distributions of the line fitting. (a) The variation of the polar angle; (b) The variation of the azimuthal angle.

The precision of the rotation axis estimation is demonstrated with five measurements to the fixed rotation axis. As shown in Table 1, the STD for the polar angle of the estimated directions is $0.0068^{\circ}$, and for the azimuthal angle is $0.0092^{\circ}$. Comparing with the STD of the line fitting, the improvement on precision of the axis estimation may come from the optimization with the surrounding line directions, as the situation shown in Fig. 7(b).

Table 1. The results of the rotation axis estimation for five measurements

\begin{tabular}{ccc}
\hline & \multicolumn{2}{c}{ Angle (in degree) } \\
\cline { 2 - 3 } & Polar & Azimuthal \\
\hline 1 & 19.5028 & 132.5789 \\
2 & 19.4927 & 132.5558 \\
3 & 19.4895 & 132.5582 \\
4 & 19.4923 & 132.5683 \\
5 & 19.4841 & 132.5666 \\
STD & 0.0068 & 0.0092 \\
\hline
\end{tabular}

Note: STD - Standard deviation

\section{CONCLUSION}

An approach for estimation of the rotation axis using machine vision to measure the auxiliary laser is proposed in this paper. An accurately calibrated binocular stereo vision sensor is established to guarantee the precision of the axis estimation. Moreover, an auxiliary laser is introduced to provide the possibility of making the related measurement away from the physical setup of the rotating object. With the assistance of the binocular sensor and the auxiliary laser, the rotation axis can be estimated with multiple lines coming from the rotation of the laser around the fixed axis whose pose has to be estimated. The polar and azimuthal angles of the rotation axis show small variations in conducted five 
measurements. It is worth to point out while the proposed approach is designed for the derotator calibration; it also has potential applications in tasks involving the axis alignment between separate physical setups.

\section{ACKNOWLEDGEMENTS}

The support of the Humboldt Research Fellowship for Postdoctoral Researchers is gratefully acknowledged. The financial supports from the Natural Science Foundation of China (NSFC) under the grant 61775121, 61675117 and that from the Sino-German Center for Research Promotion (SGCRP) under the grant GZ 1391 are also gratefully acknowledged.

\section{REFERENCES}

[1] Pérez-López, C., Santoyo, F. M., Pedrini, G., Schedin, S. and Tiziani, H. J., "Pulsed digital holographic interferometry for dynamic measurement of rotating objects with an optical derotator," Appl. Opt. 40(28), 51065110 (2001).

[2] Altmann, B., Pape, C. and Reithmeier, E., "Temperature measurements on fast-rotating objects using a thermographic camera with an optomechanical image derotator," Proc. SPIE Infrared Sensors, Devices, and Applications VII, 10404, 104040P (2017).

[3] Reithmeier, E., Mirzaei, S. and Kasyanenko, N., "Optical vibration and deviation measurement of rotating machine parts," Optoelectron. Lett. 4(1), 45-48 (2008).

[4] Rahlves, M., Mirzaei, S., Fahlbusch, T. and Reithmeier, E., "In-plane and out-of-plane deformation and vibration measurement using an optomechanical image derotator," Proc. SPIE Optical Inspection and Metrology for NonOptics Industries, 7432, 74320L (2009).

[5] Boedecker, S., Dräbenstedt, A., Heller, L., Kraft, A., Leonhardt, A., Pape, C., Ristau, S., Reithmeier, E. and Rembe, C., "Optical derotator for scanning vibrometer measurements on rotating objects," Proc. SPIE Seventh International Conference on Vibration Measurements by Laser Techniques: Advances and Applications, 6345, 63450M (2006).

[6] Mirzaei, S., Abo-Namous, O., Beichert, G., Fahlbusch, T. and Reithmeier, E., "Developing a new generation of optomechanical derotator for analysis of the dynamic behaviour of rotating components," Proc. SPIE Advances in Optomechanics, 7424, 74240I (2009).

[7] Rohloff, B., Pape, C. and Reithmeier, E., "Automated calibration of an optomechanical derotator using 6-axes parallel kinematics and industrial image processing algorithms," Opt. Eng. 53(10), 104101 (2014).

[8] Khalil, H., Kim, D., Jo, Y. and Parka, K., "Optical derotator alignment using image-processing algorithm for tracking laser vibrometer measurements of rotating objects," Rev. Sci. Instrum. 88(6), 065111 (2017).

[9] Yin, Y., Peng, X., Liu, X., Li, A. and Qu, X., "Calibration strategy of optical measurement network for large-scale and shell-like objects," Opt. Commun. 285(8), 2048-2056 (2012).

[10]Liu, X., Cai, Z., Yin, Y., Jiang, H., He, D., He, W., Zhang, Z. and Peng, X., "Calibration of fringe projection profilometry using an inaccurate 2D reference target," Opt. Lasers. Eng. 89, 131-137 (2017). 